\title{
The Influence of Chain Orientation in the Electric Behaviour of Polymer Diodes
}

\author{
Marta Ramos and Hélder Barbosa \\ Departamento de Física, Universidade do Minho, Campus de Gualtar, 4710-057 Braga, \\ Portugal \\ E-mail: marta@fisica.uminho.pt
}

\begin{abstract}
Recently some experimental results have showed that the spatial alignment of conjugated polymer chains on nanometre length scales can influence the behaviour of polymer-based electronic devices, such as light-emitting diodes, field effect transistors, and photovoltaic cells. The effects of chain orientation at electrode-polymer interfaces on the charge injection process and charge mobility through the polymer layer are not well understood. In this work we use a generalized dynamical Monte Carlo method to study the influence of different polymer chain orientation relative to the electrodes surface on the electric behaviour of single-layer polymer diode, namely density current and charge density.
\end{abstract}

\section{Introduction}

The use of conjugated polymers in electronic and optoelectronic applications goes from light-emitting diodes (LEDs) [1], to field-effect transistors (FETs) [2], and photovoltaic (PV) cells [3], among others. In all these devices, the morphology of the active polymer layer can critically influence the device behaviour $[4,5]$. The folding and bending of the polymer chains, that can be seen at the nanometre scale as a connection of conjugated segments with varied lengths, and their position and orientation with respect to neighbouring chains and/or the substrate, in which they are deposit, influences the electric properties of these polymer-based devices. However, from the experimental results [6], it is not possible to know the relation between the orientations of the polymer strands and the device electric behaviour. Most of the polymer films are prepared using the spin-cast technique that leads to polymer strands taking a preferential orientation parallel to the electrodes [7]. However, polymer chains tend to interpenetrate each other and there is the possibility of appearing domains within the polymer layer having perpendicular [8] and random [9] orientations relative to the electrodes surface.

In this work, we study the influence of the orientation of the conjugated polymer segments on the electric behaviour of single-carrier polymer diodes, using a computational model based on a generalized dynamical Monte Carlo method, which includes explicitly the nanostructure of the polymer layer and the molecular properties of the polymer as input parameters. 


\section{Device Model and Simulation Method}

In order to build the nanostructure of a polymer diode with a specific morphology, we placed straight conjugated segments of poly(pphenylenevenylene) (PPV) (considered as rigid rods) randomly in the gap between two planar electrodes, separated $100 \mathrm{~nm}$ from each other, with their axis oriented parallel, perpendicular and randomly relative to the electrodes surface. The minimum distances $(0.650 \mathrm{~nm})$ allowed between the polymer strands and between those and the electrodes, well as the mean value ( 7 monomers) of a Gaussian distribution of strand lengths, were taken from previous theoretical and experimental results reported elsewhere $[8,10]$.

In this model we consider that charge injection/collection from/by the electrodes and intermolecular charge transport within the polymer network occur by hopping with a frequency given by [10]:

$$
w_{i j}=w_{0} \times \cos \theta \times \exp \left(-\frac{r_{i j}-r_{0}}{r_{0}}\right) \times\left\{\begin{array}{c}
\exp \left(-\frac{\Delta \varepsilon_{i j}}{k_{B} T}\right), \text { for } \Delta \varepsilon_{i j}>0 \\
1, \text { for } \Delta \varepsilon_{i j}<0
\end{array}\right.
$$

where the first term represents the attempt-to-escape frequency and the following terms represent the influence of the direction of the local electric field (which is the sum of the applied electric field, the field due to charge distribution within the polymer network and the field due to electrode polarization), the hopping distance and the energy barrier height (which depends on the ionization potential and electron affinity of the involved polymer strands and their bias voltage) on the hopping process, respectively. Only the process with the highest hopping probability takes place. If the local electric field is higher than the field needed to move the injected charge along the polymer strand an intramolecular charge transport is also considered. A detailed description of the injection/collection and transport processes for ohmic contacts can be found in ref. [10, 11] and the molecular properties used in this work as input parameters were taken from ref. [12].

Our dynamic model is based on the first reaction method (FRM) [13], where a queue of increasing time steps associated to the occurrence of the all electronic processes in the polymer diode is used to follow the time evolution of the charges in the device. For each electronic process there is a waiting time given by:

$$
\tau=-\frac{\ln (x)}{w_{i j}}
$$

where $w_{i j}$ is the hopping frequency associated to an electronic process, being $x$ a random number uniformly distributed between 0 and 1 . At each computer iteration the electronic process with the smallest time of occurrence takes place and removed from the queue. This time of occurrence is then subtracted to waiting times, and new enable events are inserted in the queue. 


\section{Results and Discussion}

Using the three-dimensional model described above, we performed computational simulations of single-carrier (electrons or holes) injection and transport in polymer diodes for applied electric fields ranging from $0.3 \mathrm{MV} / \mathrm{cm}$ to $0.7 \mathrm{MV} / \mathrm{cm}$. Since the results obtained for electrons and holes are similar, we just present here the results for electrons. Fig. 1 shows the time evolution of current density for all strand orientations consider in this work. The variations in time of current density are due to stochastic time dependence of all electronic processes. An increase in the applied electric field leads to an increase of the current density especially for the parallel and random polymer strand orientations relative to the electrodes. The differences in the current density between the parallel, perpendicular and random orientations are due to the charge transport along the polymer network and the charge distribution within that network, both affecting charge injection. For the polymer layer with parallel morphology, since the number of hopping positions in a neighbouring strand is the largest on and the charge hopping occurs mainly in the same direction of the applied electric field, the charge transport is limited by the energetic disorder. Therefore, an increase in the electric field reduces the energetic barrier for hopping between neighbouring strands and as a result electrons can easily percolate along the polymer network reducing the effect of charge distribution within the polymer layer on the injection process.
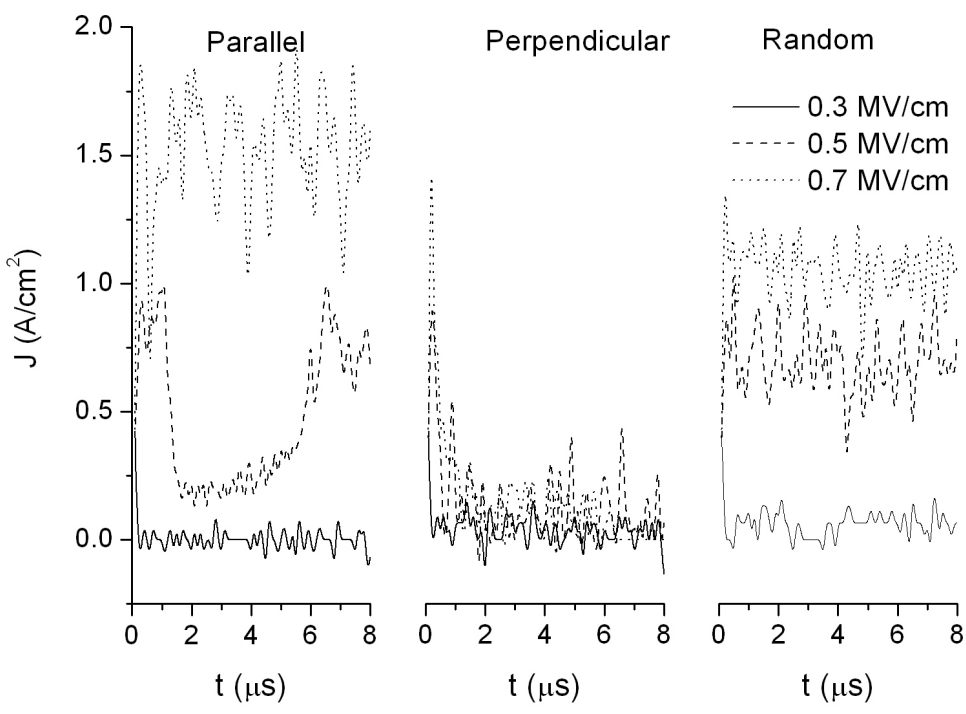

Fig. 1. Variation of the current density with time for polymer diodes with parallel, perpendicular and random chain orientations and for applied electric fields of $0.3 \mathrm{MV} / \mathrm{cm}$ (straight line), $0.5 \mathrm{MV} / \mathrm{cm}$ (dash line) and $0.7 \mathrm{MV} / \mathrm{cm}$ (dot line). 
In the case of the polymer strands oriented perpendicularly to the electrodes surface, the processes of charge injection and charge transport are strongly dependent on the morphology of the polymer layer. First, since all the strands are perpendicular to the electrodes surface, just the monomers near the electrodes are possible positions for charge injection. Second, since all the applied electric fields are smaller than the threshold for intramolecular charge mobility $(1.55 \mathrm{MV} / \mathrm{cm}$ for electrons and $2.00 \mathrm{MV} / \mathrm{cm}$ for holes) [14], injected charges in any polymer strand moves along it towards its centre, which is the position energetically more favourable. Since the hopping distance to the neighbour strands favoured by the applied electric field increases, the hopping process takes longer. As a consequence, the injected charges will stay longer near the injection electrode, limiting charge injection by changing the internal electric field. The polymer network with a random orientation of the strands, exhibits an intermediary behaviour between the parallel and the perpendicular morphologies, for the same applied electric field.
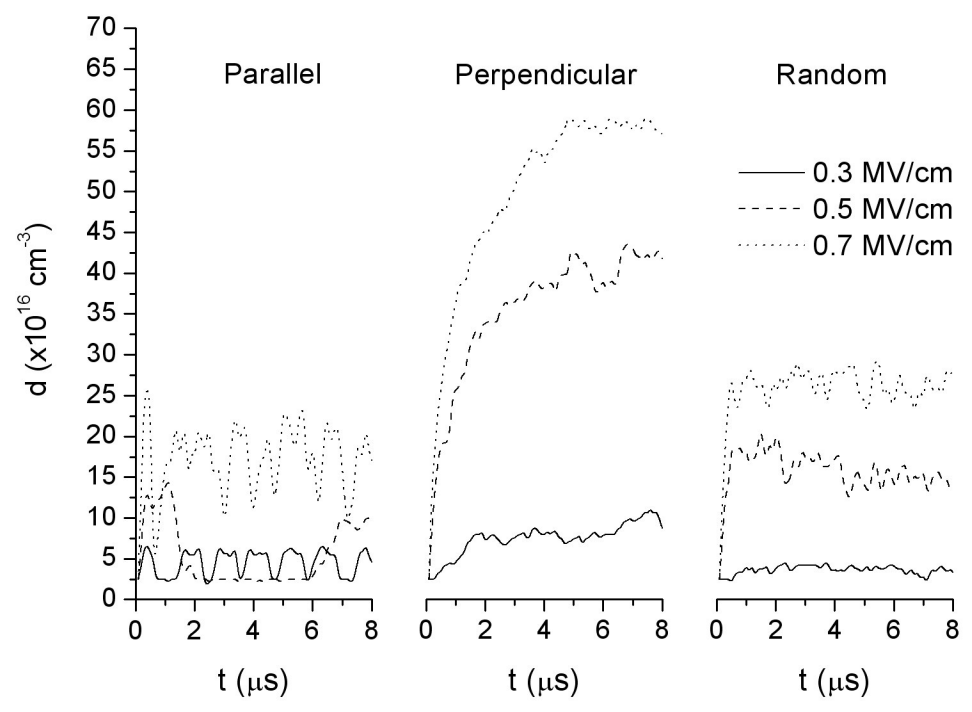

Fig. 2. Variation of the charge density with time for polymer diodes with parallel, perpendicular and random chain orientations and for applied electric fields of $0.3 \mathrm{MV} / \mathrm{cm}$ (straight line), $0.5 \mathrm{MV} / \mathrm{cm}$ (dash line) and $0.7 \mathrm{MV} / \mathrm{cm}$ (dot line).

Figure 2 shows the change in time of charge density inside the polymer layer, for all the three morphologies considered in this work. The fact the charge transport along the network is more difficult for the polymer with the perpendicular morphology allows that charges stay longer inside the polymer network increasing charge density. The opposite behaviour is predicted for the polymer layer with all strands oriented parallel to the electrodes. For all the 
polymer morphologies, an increase in the applied electric field leads to an increase in charge density due to an increase of charge injection.

\section{Conclusions}

Although our model mimics in a simple way both the nanostructure and the electronic processes involved in a single-carrier polymer diode, the values for current and charge densities obtained are comparable to those measured experimentally. Besides, it can give some insights on the effect of polymer morphology on device performance that is impossible to obtain from experiments because in a real polymer film all polymer strand orientations considered in this work, as well as the presence of physical/chemical defects and the effects of both electrode/polymer interfaces contribute to the overall current density of the device. From our results, it is clear that when the polymer segments are packed parallel to the electrodes surface there is an increase in intermolecular charge transport compared to random and perpendicular orientations which leads to an increase of current density for the same applied electric field.

Acknowledgements. This work is part of the research projects POCTI/CTM/41574/2001 and CONC-REEQ/443/EEI/2005, approved by the Portuguese Foundation for Science and Technology (FCT) and support by the European Community Fund FEDER. One of us (H.M.C.B.) is also indebted to FCT for financial support under PhD grant $N^{\circ}$ SFRH/BD/22143/2005.

\section{References}

1 B.J. Schwartz, Annu. Rev. Phys. Chem. 54, 141, 2003.

2 C. Tanase, E.J. Meijer, P.W.M. Blom, D.M. de Leeuw, Phys. Rev. Lett. 91, 21, 2003.

3 H. Hoppe, N.S. Sariciftci, Journal of Materials Chemistry 16, 1, 2006.

4 T.L. Benanti, D. Venkataraman, Photosynth. Res. 87, 1, 2006.

5 M. Jaiswal, R. Menon, Polym. Int. 55, 12, 2006.

6 J. Liu, Y.J. Shi, L.P. Ma, Y. Yang, J. Appl. Phys. 88, 2, 2000.

7 C.Y. Yang, F. Hide, M.A. Diaz-Garcia, A.J. Heeger, Y. Cao, Polymer 39, 11, 1998.

8 B.G. Sumpter, P. Kumar, A. Mehta, M.D. Barnes, W.A. Shelton, R.J. Harrison, J. Phys. Chem. B 109, 16, 2005.

9 J. Kim, J. Lee, C.W. Han, N.Y. Lee, I.J. Chung, Appl. Phys. Lett. 82, 24, 2003.

10 M.M.D. Ramos, H.M.G. Correia, J. Phys.: Condens. Matter 18, 2006.

11 H.M.C. Barbosa, M.M.D. Ramos, Plasma Process. Polym. 4, 2007.

12 A.M. Stoneham, M.M.D. Ramos, A.M. Almeida, H.M.G. Correia, R.M. Ribeiro, H. Ness, A.J. Fisher, J. Phys.-Condes. Matter 14, 42, 2002.

13 J.J. Lukkien, J.P.L. Segers, P.A.J. Hilbers, R.J. Gelten, A.P.J. Jansen, Phys. Rev. E 58, 2, 1998 .

14 A.M. Almeida, M.M.D. Ramos, H.G. Correia, Comput. Mater. Sci. 27, 1-2, 2003. 
